\title{
Conséquences locales et générales de la présence $d$ 'une dent natale chez le nouveau-né. À propos d'un cas clinique
}

\author{
Manuel Messa Savi de Tove*, Ramata Bakayoko-ly, Koffi Arthur N'guessan \\ Département d'Odonto-Stomatologie pédiatrique (OSP), UFR d'Odonto-Stomatologie, Abidjan, Côte d'Ivoire
}

(Reçu le 15 août 2013, accepté le 3 novembre 2013)

Mots clés : nouveau-né / dent natale / ulcération traumatique / dénutrition / avulsion dentaire

Résumé - Introduction : Une « dent natale » est une dent présente à la naissance. C'est un phénomène rare. La présence de ces dents peut avoir des conséquences sur la santé buccale et générale du nouveau-né. Observation : La mère d'un nouveau-né de sexe féminin de 2 semaines a consulté pour la dénutrition progressive de son enfant par refus de l'alimentation. L'enfant avait des pleurs incessants. L'examen clinique endobuccal a mis en évidence la présence d'une incisive mandibulaire natale associée à une ulcération traumatique de la face ventrale de la langue. La thérapeutique proposée a été l'avulsion. Le nouveau-né a repris une alimentation normale dans les jours qui ont suivi, les douleurs associées à la tétée ayant disparu. Son état de santé s'est amélioré. Discussion : L'origine héréditaire des dents natales a été démontrée. Les dents les plus souvent incriminées sont les incisives centrales mandibulaires. Elles sont peu développées. Leur avulsion est indiquée quand elles sont excessivement mobiles, douloureuses, susceptibles d'être ingérées et lorsqu'elles créent une ulcération de la face ventrale de la langue (syndrome de Riga-Fede).

Key words:

Abstract - Local and general consequences of presence of natal tooth in newborn. A case report. newborn / natal tooth / Introduction: A "native tooth" is defined as a present tooth in the birth. It is a rare phenomenon. We present here traumatic ulceration / the predictable consequences of this anomaly in the first days of the life. Observation: The mother of a female denutrition / tooth extraction newborn child of 2 weeks consulted for the progressive undernutrition of her child by refusal of the food. The child had ceaseless tears. The endobuccal clinical examination highlighted the presence of an incisor native mandibulaire associated with a traumatic ulceration of the lap face of the tongue. The proposed therapeutics was the avulsion. The newborn then took back a normal food in the days which followed, the pains associated with the sucking having disappeared. The health of the newborn child improved. Discussion: The hereditary origin of the native teeth has been demonstrated. Teeth most often incriminated are the central mandibular incisors Their avulsion is indicated on one hand when they are excessively mobile, painful, susceptible to be ingested and on the other hand when they create an ulceration of the ventral surface of the tongue (Riga-Fede syndrome).

\section{Introduction}

Les superstitions concernant les dents natales sont nombreuses [1, 2]. En Angleterre, les enfants nés avec cette particularité étaient destinés à devenir des soldats célèbres. Ceux nés en France et en Italie considérés comme de futurs conquérants du monde. À l'inverse, en Chine, en Pologne, en Inde et en Afrique, ces enfants étaient assimilés à des monstres et des porteurs de malheur. Indépendamment de ces croyances, les dents natales ont dans la grande majorité des cas une répercussion néfaste sur la santé du nouveau-né.

\section{Observation}

La mère d'un nouveau-né de sexe féminin âgé de 2 semaines a consulté pour l'altération progressive de l'état général de sa fille du fait de son refus de s'alimenter. Cette enfant présentait une diminution du tonus général, un amaigrissement important, une pâleur cutanée et avait des pleurs incessantes. L'accouchement s'était déroulé sans particularité. Le nouveauné n'avait ni fièvre, ni adénopathie, écartant à priori une origine infectieuse. L'examen de la cavité buccale a mis en évidence la présence d'une incisive natale mandibulaire mobile.

\footnotetext{
*Correspondance : savi_manuel2006@yahoo.fr
} 


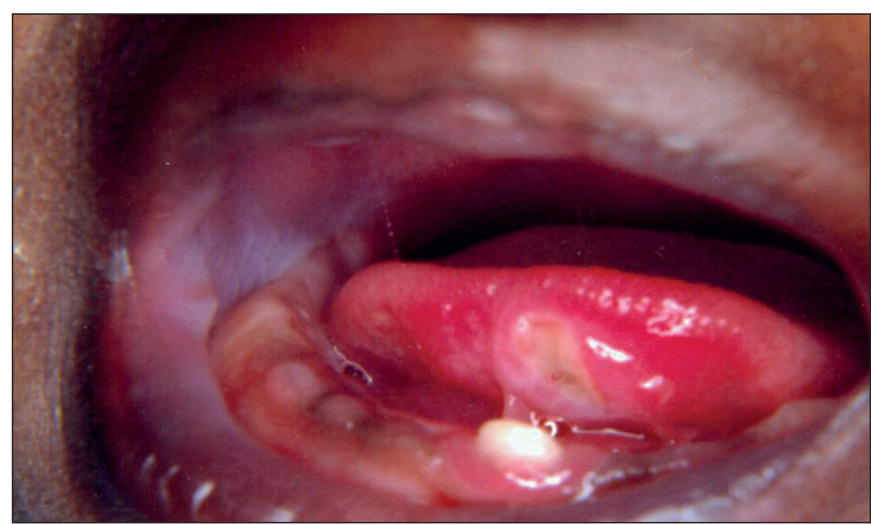

Fig. 1. Présence d'une incisive mandibulaire natale à l'origine d'une ulcération traumatique de la face ventrale de la langue.

Fig. 1. Presence of a native mandibular incisor causing a traumatic ulceration of the ventral surface of the tongue.

En regard de la dent, une ulcération de la face ventrale de la langue était présente. Le diagnostic de syndrome de Riga-Fede due à une dent natale a été posé (Fig. 1). La mobilité de la dent était telle qu'il existait un risque d'inhalation. Aucun examen radiographique n'a pu être proposé en raison des difficultés à réaliser un cliché dans de bonnes conditions. La décision d'extraire cette dent a été prise. Son avulsion a été réalisée sous anesthésie locale, sans difficulté, la dent extraite ne présentait pas de structure radiculaire (Fig. 2). Le contrôle à une semaine a confirmé que les difficultés de tétée avaient disparu. L'enfant avait repris du poids et ne présentait plus d'altération de l'état général. L'ulcération linguale avait également quasiment disparu.

\section{Discussion}

Les dents présentes dans la cavité buccale à la naissance sont appelées « dents natales ». Celles qui font leur éruption durant les 30 premiers jours de la vie sont dites « dents néonatales ». Les dents natales sont plus fréquemment observées que les dents néo-natales et près de $90 \%$ de ces dents sont des dents temporaires ayant fait une éruption précoce. La prévalence des dents natales varie de $1 / 2000$ à $1 / 3000$, avec une forte variation raciale et un taux maximal de $8,8 \%$ chez les Indiens de l'Alaska [3, 4]. La prévalence est légèrement plus importante chez les filles. Les dents natales sont rares chez les enfants prématurés. L'infection, les états fébriles, les traumatismes, la malnutrition, la position superficielle du germe de la dent, la stimulation hormonale et l'exposition maternelle à des toxines environnementales [5] ont été évoqués comme facteurs de causalité. Seule l'origine héréditaire a pu être démontrée [6-9]. Elles sont également présentes dans certains syndromes malformatifs [8, 10, 11]. Les dents natales sont présentes chez $2 \%$ des nourrissons présentant une fente

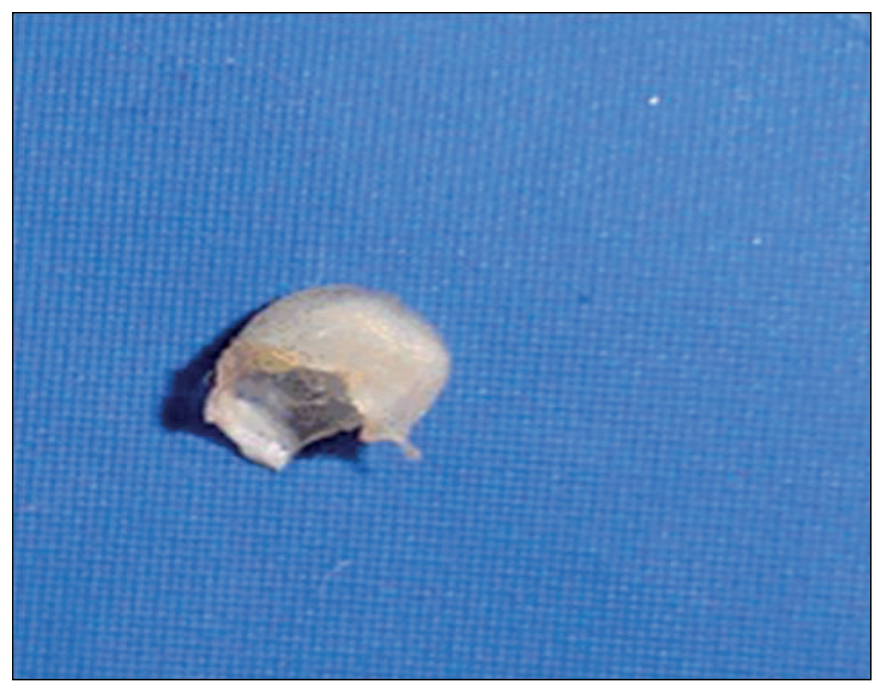

Fig. 2. Dent natale extraite. Notez l'absence complète de structure radiculaire.

Fig. 2. Extracted native tooth. Note the complete absence of root structure.

labio-palatine unilatérale et chez $10 \%$ de ceux présentant une fente bilatérale. La taille et la forme d'une dent natale ressemblent à celles d'une dent temporaire, mais la dent natale est habituellement plus petite, conique avec une couleur jaune ou marron opaque. Elles sont peu développées, avec peu ou pas de formation radiculaire $[1,3]$. Les dents les plus souvent incriminées sont les incisives centrales mandibulaires (85\%), suivies par les canines et molaires mandibulaires $(3 \%)$, les incisives centrales maxillaires (1\%), puis les canines et molaires maxillaires (moins de $1 \%$ ). Moins de $10 \%$ des dents natales sont surnuméraires [12]. Histologiquement, la majorité des dents natales ont un émail dysplasique et sont hypominéralisées. La dentine est irrégulière, avec présence d'ostéodentine dans la portion cervicale et de dentine globulaire dans la région coronaire. Leur bord incisif peut aussi être hypominéralisé [4]. Les nodules de Bohn et les perles d'Epstein [13, 14] peuvent être confondus avec une dent natale. Si une dent natale est asymptomatique aucun traitement n'est nécessaire. La présence d'une ulcération de la face ventrale de la langue n'est pas suffisante pour indiquer une avulsion [15-19]. Le traitement proposé est le lissage du bord incisif ou la mise en place d'une résine composite sur le bord incisif [15-19]. Dans la grande majorité des cas, l'avulsion est nécessaire car la dent est mobile, douloureuse, susceptible d'être ingérée ou inhalée. Dans quelques cas, on a rapporté une lacération traumatique des mamelons de la mère après la tétée. Cette donnée est néanmoins controversée car, pendant l'allaitement, la langue du nouveau-né s'interpose entre les dents natales et le mamelon [15-19]. Dans le cas présenté, l'altération de l'état général et la mobilité de la dent natale ont justifié l'avulsion. 


\section{Conclusion}

La présence d'une dent natale est rare. Les incisives mandibulaires sont les plus fréquentes. Leur avulsion n'est pas systématique. Elle est indiquée s'il existe un risque d'inhalation et/ou une symptomatologie douloureuse ayant une répercussion sur l'alimentation.

\section{Conflits d'intérêt : aucun}

\section{Références}

1. Sureshkumar R, Mc Aulay AH. Natal and neonatal teeth. Arch Dis Child Neonatal 2002;87:227-30.

2. Maheswari NU, Kumar BP, Karunakaran, Kumaran ST. “Early baby teeth" Folklore and facts. J Pharm Sci Bioallied 2012;4:329-33.

3. Sothinathan R, Shakib K. Natal teeth: a sign of fortuity or grave misfortune. Dent J 2011;210:265-6.

4. Baumgart M, Lussi A. Dents natales et néonatales. Rev Mens Suisse Odontostomatol 2006;116:904-9.

5. Alaluusua S, Kiviranta H, Leppaniemi A. Natal and neonatal teeth in relation to environmental toxicants. Pediatr Res 2002;52: 652-5.

6. Bodenhoff J. Natal and neonatal teeth. Dent Abstr 1960;5: 485-6.

7. Chow MH. Natal and neonatal teeth. JADA 1980;100:215-6.
8. Cunha R, Carrilho Boer F, Torriani DD. Natal and neonatal teeth: review of literature. Pediatr Dent 2001;23:158-62.

9. Hals E. Natal and neonatal teeth. Histological investigation in two brothers. Oral Surg Oral Med Oral Pathol 1957;10:509-21.

10. Kimoto S, Suga H, Yamaguchi M, Uchimura N, Ikeda M, Kakizawa T. Hypoplasia of primary and permanent teeth following osteitis and the implications of delayed diagnosis of a neonatal maxillary primary molar. Int J Peadiatr Dent 2003;13:35-40.

11. Kates $G$, Needleman H, Holmes LB. Natal and neonatal teeth: a clinical study. JADA 2004;109:441-43.

12. Rao RS, Mathad SV. Natal teeth: case report and review of literature. J Oral Maxillofac Pathol 2009;13:41-6.

13. Leung AK, Robson WL. Natal teeth: a review. J Natl Med Assoc 2006;98:226-28.

14. Willies-Jocobo LJ, Isaacs H Jr, Stein MT. Pyogenic granuloma presenting as a congenital defect epulis. Arch Pediatr Adolesc Med 2000;154:603-5.

15. Ceyhan AM, Yildirim M, Basak PY, Akkaya VB, Ayata A. Traumatic lingual ulcer in a child: Riga-Fede disease. Clin Exp Dermatol 2009;34:186-88.

16. Baroni A, Capristo C, Rossiello L, Faccenda F, Satriano RA. Lingual traumatic ulceration (Riga-Fede disease). Inter $\mathrm{J}$ Dermatol 2006;45:1096-97.

17. Hegde RJ. Sublingual traumatic ulceration due to neonatal teeth (Riga-Fede disease). J Indian Soc Pedo Prev Dent 2005;23:51-2.

18. Jariwala D, Graham RM, Lewis T. Riga-Fede disease. Br Dent J 2008;204:171.

19. Choi SC, Park JH, Choi YC, Kim GT. Sublingual traumatic ulceration (a Riga-Fede disease): report of two cases. Trauma Dent 2009;25(3):48-50. 